Check for updates

Cite this: Mater. Adv., 2022, 3,2070

Received 16th December 2021, Accepted 29th December 2021

DOI: 10.1039/d1ma01190a

rsc.li/materials-advances

\title{
Emissive organogel mediated construction of a flexible covalent organic polymer for the separation of aniline for water purification $\dagger$
}

\begin{abstract}
Sayan Maiti, Tapas Ghosh, (D) Arati Samal and Apurba K. Das (D) *
A flexible covalent organic polymer (COP) has been successfully synthesized via dynamic covalent gel (DCG) formation through an imine condensation reaction between 6-hydrazinonicotinic hydrazide hydrate and benzene-1,3,5-tricarboxaldehyde within $7 \mathrm{~min}$ under ambient conditions. An emissive organogel mediated protocol has been developed for the construction of an amorphous polymer (COP), selectively in $\mathrm{N}, \mathrm{N}$-dimethyl acetamide (DMA). Interestingly, two non-emissive building block units without large $\pi$-conjugated structures have been engaged for the construction of green emissive COP-gel. The green emission of COP-gel is generated by the intermolecular $\mathrm{H}$-bonding assisted aggregation induced emission phenomenon. The dried COP efficiently adsorbs aniline molecules into its cavities and separates them from binary mixtures of aniline/nitrobenzene and aniline/water, respectively.
\end{abstract}

\section{Introduction}

Covalent organic polymers (COPs) are a unique class of organic porous materials that are constructed covalently from organic subunits. ${ }^{1}$ Mostly, COPs are constructed from functional organic building blocks through the formation of $\mathrm{B}-\mathrm{N}, \mathrm{B}-\mathrm{O}$, $\mathrm{C}=\mathrm{N}, \mathrm{C}=\mathrm{C}, \mathrm{C}-\mathrm{N}, \mathrm{C}=\mathrm{N}, \mathrm{C}=\mathrm{N} / \mathrm{C}-\mathrm{O}, \mathrm{N}=\mathrm{N}$, and $\mathrm{Si}-\mathrm{O}$ bonds. ${ }^{2,3}$ Several methods or templates have been developed to synthesize COPs. ${ }^{4-6}$ However, it is still a difficult task to develop a synthetic approach for the synthesis of large-scale COPs under ambient conditions. In order to extend the implementation of porous organic polymers, a time-efficient synthetic method is being sought. An aggregation assisted synthetic procedure can be the easiest and alternative route for the scalable synthesis of COPs. Covalent dynamic gel formation can be an intermediate aggregated state that can be used for the large scale synthesis of COPs. On the other hand, it is a challenging task to evaluate the nature of covalent dynamic gels despite their various applications in catalysis, sensors and adsorbent materials. ${ }^{7-10}$

Recently, covalent organic polymers $(\mathrm{COPs})^{11,12}$ have received considerable importance in membrane technologies due to their selective molecular adsorption and separation properties. ${ }^{13-18}$ The characteristics of COPs can be altered at the atomic level by modifying the synthetic procedures and suitable monomers. Functionalized Schiff base covalent organic polymers as membrane materials exhibit high efficiency in the separation of

Department of Chemistry, Indian Institute of Technology Indore, Khandwa Road, Indore 453552, India. E-mail: apurba.das@iiti.ac.in

$\dagger$ Electronic supplementary information (ESI) available. See DOI: 10.1039/d1ma01190a molecules/ions. ${ }^{19-23}$ In this regard, the introduction of various functional groups into porous materials has been reported as an effective way to improve their adsorption capacity and selectivity towards organic molecules. The efficient separation of aromatic organic solvents from solvent mixtures and their derivatives, which are critical components of the chemical industry, can lead to higher reusability.

An organic solvent like aniline is a crucial intermediate in the production of medicines, rubber accelerators and antiaging chemicals. ${ }^{24}$ Moreover, aniline is a common organic contaminant with high toxicity, rapid bio-enrichment and challenging biodegradability. ${ }^{25}$ Since aniline poses potential danger in industrial scale separation, and threat to human health and the environment due to its high toxicity, volatility or flammability, there is a strong demand for the development of highly effective and environmentally safe materials for the separation of aniline from solvent mixtures. ${ }^{26,27} \mathrm{~A}$ wide range of methods and technologies have been developed to remove aniline. ${ }^{28,29}$ On the other hand, several nitrogen atoms containing recyclable green switchable solvents are widely used for the extraction or separation of organic solvents. ${ }^{30,31}$ However, most of the switchable solvents are amines that separate the desired product from the solvent mixtures through a phase transition process. Li et al. used nitrogen containing ionic liquids for the separation of binary mixtures by extractive distillation methods. ${ }^{32}$ The extractive distillation method can also be used to separate organic solvent mixtures. ${ }^{33,34}$ The above-mentioned methods or processes are quite complicated and are characterized by high energy consumption and high equipment cost, therefore, it is necessary to develop a convenient method and 

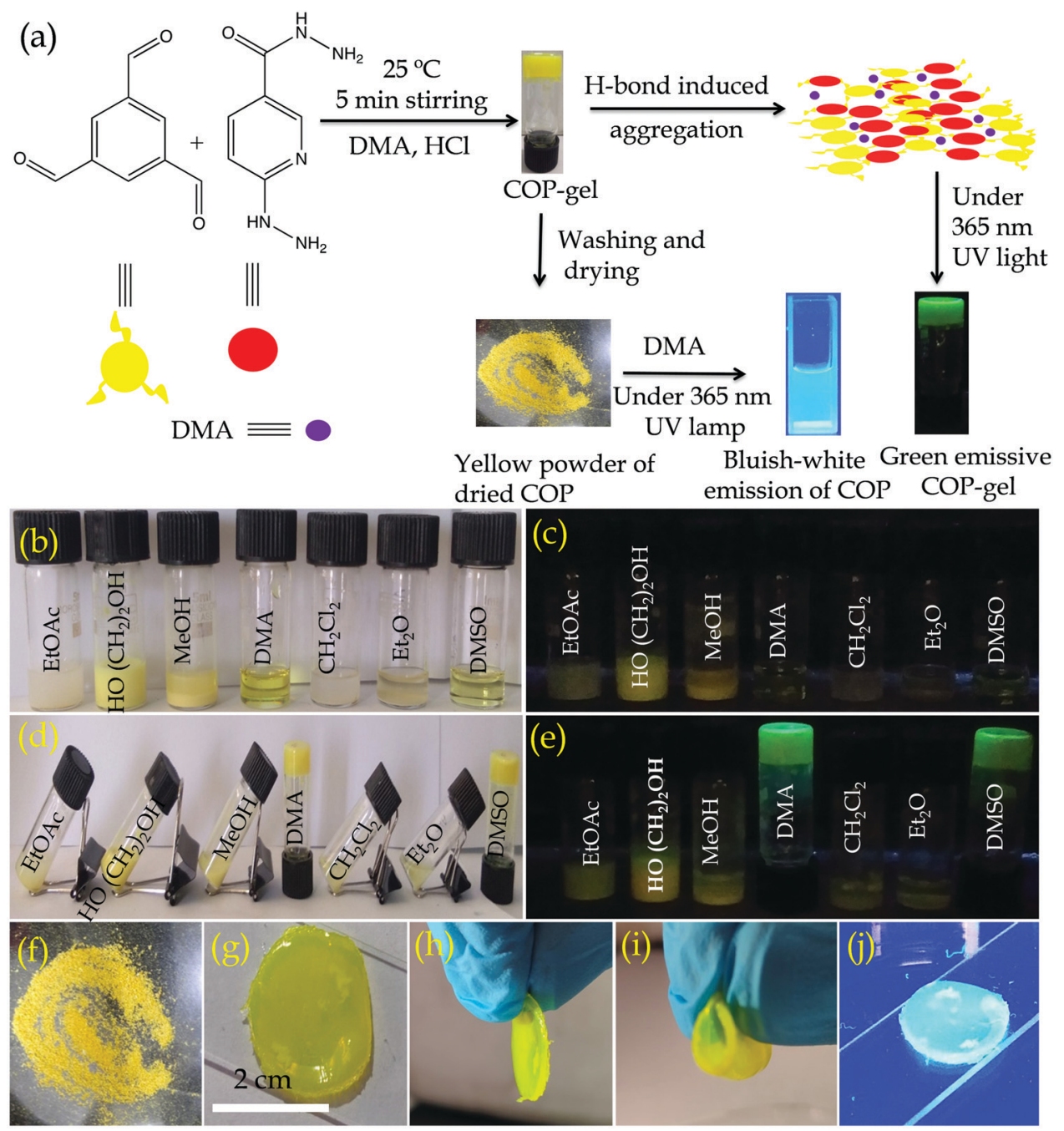

Fig. 1 (a) Schematic representation of porous COP synthesis, and aniline separation and adsorption by COP. Solution mixture of 6-hydrazinonicotinic hydrazide hydrate and benzene-1,3,5-tricarboxaldehyde in different solvents (b) in day light and (c) under $365 \mathrm{~nm}$ UV light. Solution mixture of 6-hydrazinonicotinic hydrazide hydrate and benzene-1,3,5-tricarboxaldehyde in different solvents after the addition of $1 \mathrm{~N} \mathrm{HCl}$ (d) in day light and (e) under $365 \mathrm{~nm}$ UV light. Optical images of (f) COP powder, ( $\mathrm{g}$-j) films formed with COP and (i) COP film (shows white-cyan colour emission) under $365 \mathrm{~nm}$ UV light.

material with lower equipment costs for the separation of organic solvents. In this regard, adsorption using solid adsorbents is a quick and easy method, and nitrogen functionalized COPs can be innovative solid adsorbent materials with high adsorption capacity and selectivity for the separation of organic solvents. The adsorption technique is demonstrated to be enhanced with better adsorption capacity and selectivity due to the possible H-bonding, and electrostatic and acid-base interactions between the surface functional groups on the COPs and adsorbates. Herein, our objectives are (1) to develop new synthetic methodologies with large-scale synthesis of functionalized COP materials under ambient conditions, (2) to develop cavities inside the COP backbone through the dynamic covalent gel (DCG) strategy and (3) to investigate the intrinsic properties of functionalized COP materials. In this work, we have synthesized hydrazine, hydrazide and pyridine group functionalized COP via emissive organogel formation within 7 min (Fig. 1a).
The gel formation plays a key role in synthesizing the COP on a large scale. The COP is capable of adsorbing or encapsulating aniline selectively in its cavities and selectively separates it from binary mixtures of aniline/nitrobenzene and aniline/water.

\section{Results and discussion}

Here we have developed a facile synthetic strategy to synthesize covalent organic polymers with 6-hydrazinonicotinic hydrazide hydrate and benzene-1,3,5-tricarboxaldehyde as building block units. The COP has been synthesized via emissive organogel formation in DMA under acidic conditions within 7 min (Fig. 1a) under ambient conditions. The gel is formed with a COP concentration of about $0.038-0.040 \mathrm{~mol} \mathrm{~L}^{-1}$. Among ethyl acetate (EtOAc), diethyl ether $\left(\mathrm{Et}_{2} \mathrm{O}\right)$, ethylene glycol $\left(\mathrm{CH}_{2} \mathrm{OH}\right)_{2}$, methanol $(\mathrm{MeOH})$, $\mathrm{N}, \mathrm{N}$-dimethyl acetamide (DMA), dichloromethane $\left(\mathrm{CH}_{2} \mathrm{Cl}_{2}\right)$ and 
dimethyl sulfoxide (DMSO), the COP was successfully formed in DMA via emissive gel formation (Fig. 1). Fig. S1 (ESI $\dagger$ ) shows the 3D block representation of COP-gel. To obtain pure COP, the COP-gel was dried and washed several times with water, ethyl acetate, $\mathrm{N}, \mathrm{N}$ dimethyl formamide, and methanol to remove unusual impurities. After drying at $90{ }^{\circ} \mathrm{C}$, the COP was characterized using several techniques.

The FT-IR spectrum of COP demonstrates the successful construction of COP via the formation of $-\mathrm{C}=\mathrm{N}$ bonds. ${ }^{35-37}$ The peaks at 1530 and $1605 \mathrm{~cm}^{-1}$ are assigned to $-\mathrm{C}=\mathrm{C}-$ and $-\mathrm{C}=\mathrm{N}$ bond stretching. A peak at $1650 \mathrm{~cm}^{-1}$ appears due to the $-\mathrm{C}(=\mathrm{O}) \mathrm{NHNH}^{-}$stretching band (Fig. 2a). The powder XRD pattern of COP suggests that the polymer is successfully constructed from two crystalline secondary building block units (Fig. 2b). The broad powder XRD pattern of COP exhibits its amorphous polymeric nature (Fig. 2b). A slight broad peak at higher $2 \theta$ indicates the strong $\pi-\pi$ stacking interaction between the COP layers. To investigate the thermal stability of COP, we have performed thermogravimetric analysis (Fig. 3a). The COP exhibits high thermal stability. The TGA spectrum of COP shows a $4 \%$ weight loss in the lower temperature range from 27 to $230{ }^{\circ} \mathrm{C}$ due to the evaporation of adsorbed moisture in COP, followed by weight losses of around $6.34 \%$ and $15.95 \%$ over the temperature range of 230 to $370{ }^{\circ} \mathrm{C}$ and 370 to $402{ }^{\circ} \mathrm{C}$, respectively. Following that, a gradual loss of weight is observed as the temperature increases.

The solid-state ${ }^{13} \mathrm{C}$ CP-MAS NMR spectrum of COP also supports the successful formation of COP (Fig. 3b). The peaks from 120-140 ppm confirm the presence of phenyl groups. The peak at 159 ppm suggests acyl hydrazone groups in COP. The peak at 163 ppm confirms the presence of carbonyl groups of $-\mathrm{C}(\mathrm{O}) \mathrm{NHNH}-$ and a peak at $150 \mathrm{ppm}$ appears due to the $\mathrm{C}=\mathrm{N}$ bond of the pyridine ring. ${ }^{35}$

The porosity of COP was evaluated by performing BET surface area analysis by $\mathrm{N}_{2}$ sorption at $77 \mathrm{~K}$ and 1 bar pressure. No hysteresis loop is observed in the isotherm. The COP shows a type II reversible isotherm (Fig. S2, ESI $\dagger$ ). The presence of micropores is demonstrated by a steep uptake at a low relative pressure $P / P_{0}$. This result clearly suggests that microporosity has been observed inside the COP during the gel formation. At high relative pressures of $P / P_{0}>0.9$, a significant volume of $\mathrm{N}_{2}$ adsorption is observed. The increase in $\mathrm{N}_{2}$ adsorption at $P / P_{0}>$ 0.9 and the complete absence of saturation in the adsorption isotherm can be ascribed to the condensation of $\mathrm{N}_{2}$ gas molecules in inter-particular voids or in larger pores. The calculated BET surface area of COP is $49.69 \mathrm{~m}^{2} \mathrm{~g}^{-1}$. The pore size distribution of COP was studied via the NLDFT method (Fig. S3, ESI $\dagger$ ). The calculated pore volume of COP is

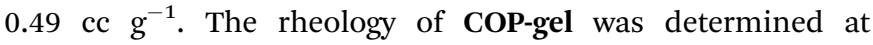
ambient temperature to evaluate the mechanical properties of COP-gel (Fig. 3c and d). The oscillatory frequency sweep test was performed in the frequency range of 0.05 to $100 \mathrm{rad} \mathrm{s}^{-1}$ at an applied strain of $1 \%$. The storage modulus $\left(G^{\prime}\right)$ is significantly higher than the loss modulus $\left(G^{\prime \prime}\right)$ over the studied frequency range which clearly indicates the viscoelastic and robustness nature of COP-gel. According to scanning (SEM) and high resolution transmission electron microscopy (HRTEM) images, COP generates a sponge-like porous structure made up of interconnected nanoscale spherical particles (Fig. 4a and b). The particles with a diameter of 20-40 nm combine to form a three-dimensional gel matrix. The amorphous nature of COP is also supported by its selected area diffraction pattern (SAED) pattern (Fig. 4b, inset). The surface hydrophobicity and surface roughness of COP were evaluated using atomic force microscopy (Fig. 4c). The nano-level surface roughness illustrates the hydrophobicity of COP. In general, highly hydrophobic materials have a tendency to aggregate into spherical assembly upon coating on a glass surface. ${ }^{38}$ Similarly, the COP also shows this salient feature. The COP surface contains several crests and troughs which have been observed from the 3D view (Fig. 4d). The peaks in the region of $0.5 \mu \mathrm{m}$ indicate the existence of air pockets between the peaks (valley regions). These trapped air pockets in the valley region create the hydrophobic pores in COP.

The COP-gel shows green emission within the range of 520-550 nm. Interestingly two secondary building block units
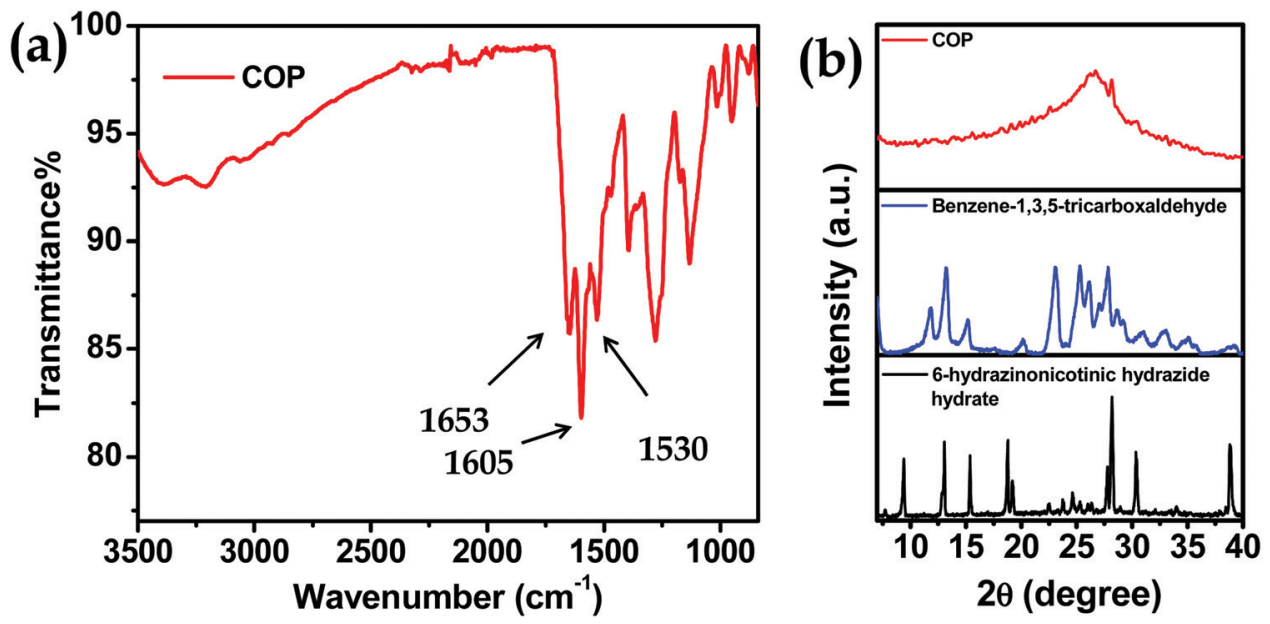

Fig. 2 (a) FT-IR spectrum of COP. (b) Powder XRD pattern of COP. 

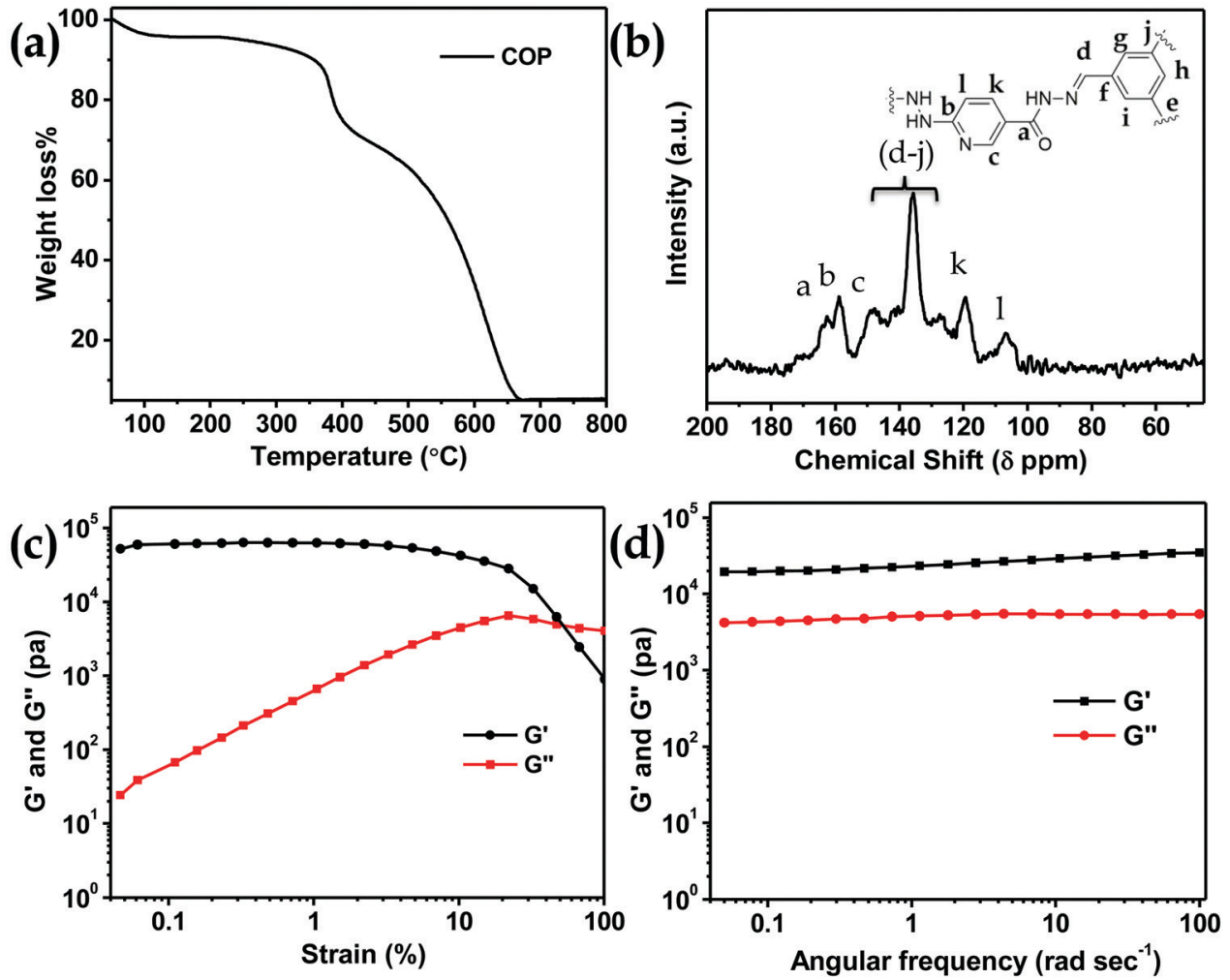

Fig. 3 (a) TGA curve of COP. (b) Solid state ${ }^{13} \mathrm{C}$ NMR spectrum of COP. Linear viscoelastic (LVE) properties of (c) COP-gel and (d) the dynamic frequency sweep experiment of COP-gel
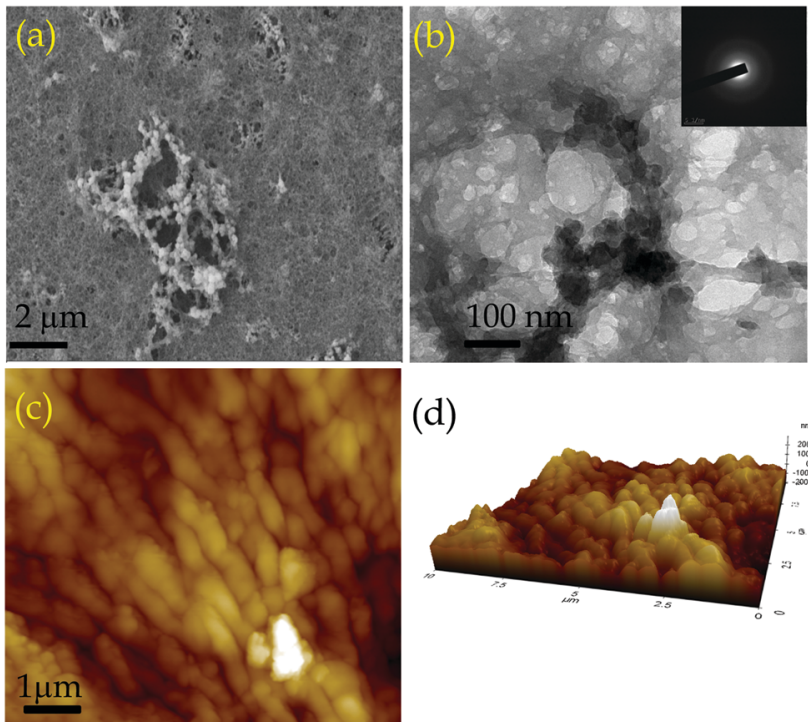

(d)

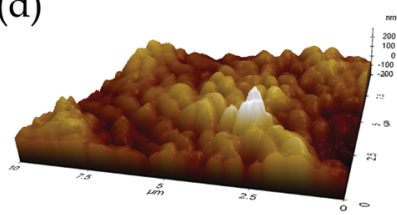

Fig. 4 (a) SEM and (b) HRTEM images of COP (Inset: SAED pattern of COP). (c) AFM image of COP and (d) 3D AFM (Z-axis interpretation) image of COP.

are non-fluorescent in the DMA solvent and the pyridine moiety is itself a fluorescence quencher. The COP backbone is rigidified through a series of $\mathrm{H}$-bonding interaction between the hydrazide groups and the solvent molecule (DMA) (Fig. S4,
ESI $\dagger$ ). These H-bonds are highly responsible for the restriction of intramolecular rotation, which causes aggregation-induced emission (AIE) ${ }^{39,40}$ The emission colour of COP originates from the distinct level of aggregation in the solvent which alters the electronic distribution and shows different fluorescent colours. So, H-bonding plays a significant role in the evolution of emissive COP-gel. By varying the concentration of building block units, we have also prepared COP through flexible thin film preparation in the DMA solvent. Different extents of aggregation occur in gel and thin films, leading to different emission colours (Fig. 1g and j). We have conducted a spectroscopic study in detail to prove that the H-bonding induced strong aggregation in COP-gel. The UV-vis spectrum of COP-gel shows an absorption maximum centered at $311 \mathrm{~nm}$, which is attributed to the $n-\pi^{*}$ transition (Fig. S5, ESI $\dagger$ ). On the other hand, an absorption peak at $423 \mathrm{~nm}$ of COP-gel is observed, which suggests the formation of strong intermolecular $\mathrm{H}^{-}$ bonds. This phenomenon indicates its higher order aggregated state by restricting intramolecular rotation (RIR) and lowering the HOMO-LUMO energy gap. ${ }^{41,42}$ Also, a broader absorption band reveals a higher order aggregated form of COP-gel (Fig. S5, ESI $\dagger$ ). The COP-gel shows dual emission at $530 \mathrm{~nm}$ with a shoulder peak at $626 \mathrm{~nm}$ (Fig. S6, ESI $\dagger$ ). We have also performed temperature dependent fluorescence studies of COP-gel. The temperature-dependent fluorescence studies reveal that the emission intensity decreases gradually with the increase in temperature (Fig. S7, ESI $\dagger$ ). These results also 
support the existence of H-bonding inside the COP-gel. The excitation spectrum of COP-gel at $530 \mathrm{~nm}$ is similar to the excitation spectrum of COP-gel at $626 \mathrm{~nm}$ (Fig. S8, ESI $\dagger$ ). These results indicate that the origin of the shoulder band at around $626 \mathrm{~nm}$ of COP-gel is assigned to the protonated state. ${ }^{43}$ An emission peak at $626 \mathrm{~nm}$ originates from the protonated form of the COP-gel (due to formation of $\mathrm{PyH}^{+}$and imine $-\mathrm{C}=\mathrm{NH}^{+}-$). The green colour emission of COP-gel is also visualized under a confocal microscope with an excitation wavelength of $405 \mathrm{~nm}$ and emission collection of $540 \mathrm{~nm}$, respectively. The confocal image of COP is shown in Fig. S9 (ESI $\dagger$ ). The confocal images further support the AIE phenomenon in the solid state of COP in the green colour region. ${ }^{44}$ We have further investigated the photophysical properties of disperse solutions of COP powder in DMA solution. As previously stated, the introduction of hydrazide functionality is crucial for strengthening the COP's emissive behaviour via strong intra- and interlayer H-bonding interactions. In the UV-vis spectra of COP solution, a broad absorption maximum is found across the wavelength range of 300 to $400 \mathrm{~nm}$, which correlates with the $n-\pi^{*}$ transitions and also reveals its aggregated state (Fig. S10, ESI $\dagger$ ). For an excitation wavelength of $340 \mathrm{~nm}$, the emission spectrum of COP in DMA shows a maximum at $474 \mathrm{~nm}$ (Fig. S10, ESI $\dagger$ ). Pyridine and hydrazide functionalized COP showcased adequate thermal and chemical stability due to the presence of several heteroatoms in the polymeric backbone, and H-bonding controlled fluorescence properties in DMA solution. The abundant heteroatoms form intra- and intermolecular $\mathrm{H}$-bonds, causing aggregation, limiting intermolecular rotation, and emitting light. The emission of COP in DMA solution is clearly attributed to the intermolecular and intermolecular H-bonding assisted aggregation induced emission properties.

The solvent extraction method is widely used to isolate aromatic organic solvent mixtures and their derivatives. The choice of material is highly influenced by its similarity to the target product, cost and safety. Nitrogen-rich switchable materials, a class of recyclable green materials, can be used to separate the organic solvent mixtures efficiently in a costeffective manner. ${ }^{45}$ Herein, the COP acts as an efficient stationary phase for the separation of organic solvents from binary organic solvent mixtures (Fig. 5 and Fig. S11, ESI $\dagger$ ).

We have taken aniline/nitrobenzene as a model organic solvent mixture for the separation experiment using dried COP. Initially, we loaded $60 \mathrm{mg}$ of COP inside a $1 \mathrm{~mL}$ $(5.5 \mathrm{~cm})$ glass syringe and the packing length was $0.15 \mathrm{~mL}$ $(0.7 \mathrm{~cm})$. At first, we took $50 \mu \mathrm{L}$ of each solvent and diluted it with hexane and passed through the COP loaded in the $1 \mathrm{~mL}$ glass syringe. The COP selectively adsorbs and separates aniline from nitrobenzene. Hexane was used as the mobile phase solvent. All the filtrates were characterized by high performance liquid chromatography (HPLC) (Fig. S12 and S13, ESI $\dagger$ ). Generally, separation of organic solvents depends on the surface charge of the COP, pore size, functionality on the COP surface and electrostatic interactions between COP and organic solvents. Here, the COP exhibits a hydrazide and pyridinic group rich surface with an average pore size distribution of 1.3 to $10 \mathrm{~nm} .{ }^{45-47}$ Aniline shows high binding interactions and easily stack with the COP backbone through $-\mathrm{NH} \cdots \mathrm{N}$ - interactions that also induce the aggregation properties of COP and reside in the pore cavity of the polymer. ${ }^{48-51}$ Nitrobenzene, on the

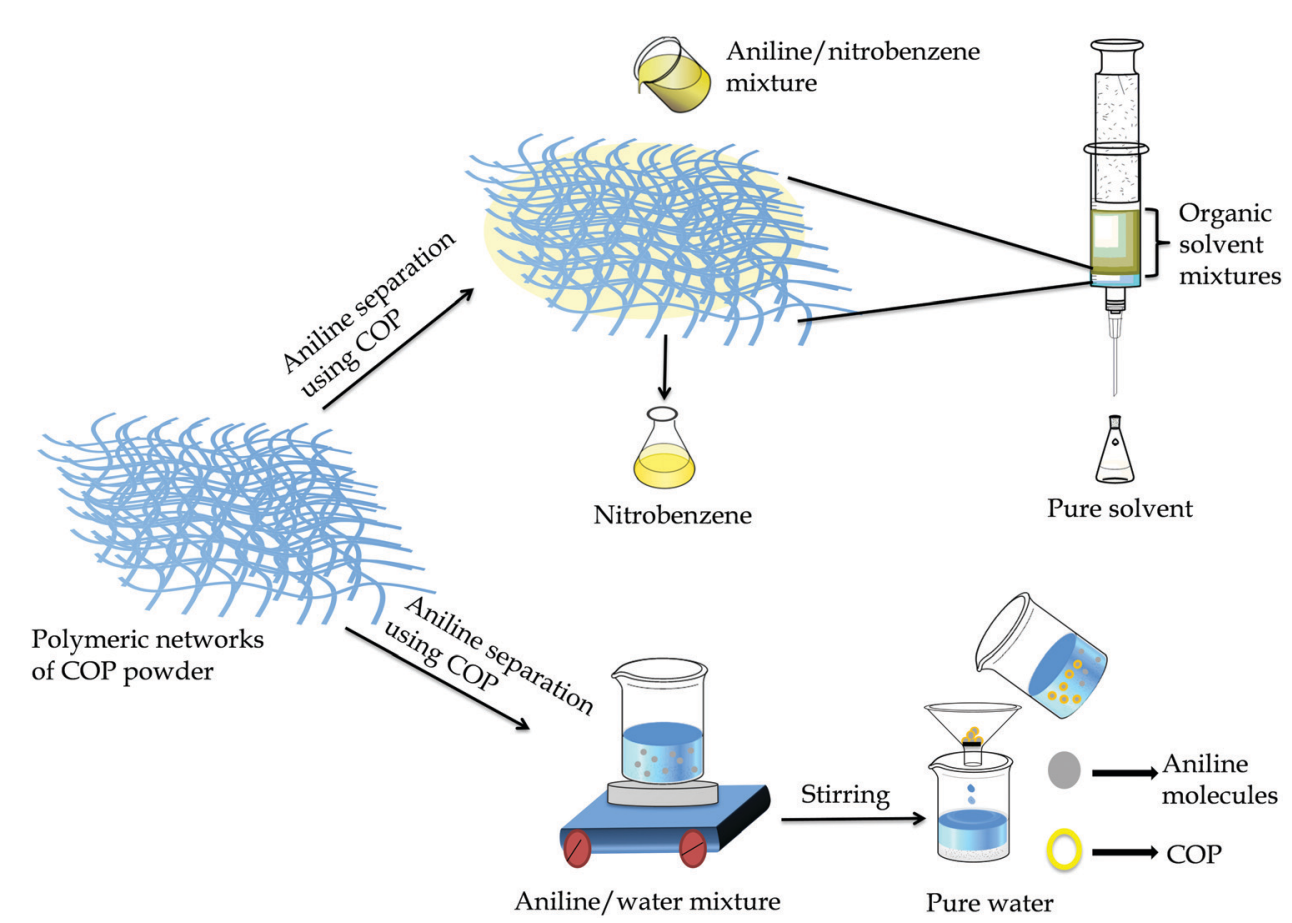

Fig. 5 Schematic representation of aniline separation from binary solvent mixtures aniline/nitrobenzene and aniline/water. 
other hand, lacks any polar amine $\left(-\mathrm{NH}_{2}\right)$ group and is unable to bind with the COP backbone. As a result, nitrobenzene can easily escape via the COP. Hexane $(6 \mathrm{~mL})$ is required to remove the entire amount of nitrobenzene. We have also investigated the adsorption limit of aniline using $60 \mathrm{mg}$ COP powder. The high adsorption limit of COP is found to be $55 \mu \mathrm{L} / 60 \mathrm{mg}$ for aniline. After each cycles, the loaded COP powder (stationary phase) was washed very well with water, ethyl acetate and methanol to remove the adsorbed solvent (aniline) from the pores of COP. After that the used COP was dried below $90{ }^{\circ} \mathrm{C}$ and used for the next cycle. The COP was reused and the COP retained its activity after five cycles (Fig. S14, ESI $\dagger$ ). For the purpose of extraction of aniline from the aniline/water mixture, we have directly used the resultant COP powder. The adsorption mechanism was followed to extract aniline where COP acts as an adsorbent for aniline in aqueous solution. Here, $45 \mathrm{mg}$ of COP powder was added to a $3 \mathrm{~mL}$ aqueous solution of aniline with a concentration of $222 \mathrm{mg} \mathrm{L}^{-1}$ and allowed to stir at $25^{\circ} \mathrm{C}$. The UV-Vis spectra of the resultant solution were recorded at different time intervals. The gradual decrease in aniline absorbance suggests quick adsorption performance (Fig. S15a, ESI $\dagger$ ). The COP exhibits significant adsorption performance for aniline, removing $83.5 \%$ of aniline in $8 \mathrm{~h}$ and achieves adsorption equilibrium (using eqn S1, ESI $\dagger$ ). The removal rate of COP for aniline is substantially higher, implying that the $-\mathrm{NH}_{2}$ group of aniline plays a crucial role in the adsorption process towards COP functionalities. Compared with nitrobenzene, COP is a better adsorbent material with fast adsorption for aniline, which is due to the well-defined porous structure and surface functionalities. The adsorption process demonstrates that the incorporated hydrazide groups of COP are favourable for adsorption due to possible H-bonding and acid-base interactions.

\section{Conclusions}

In summary, we synthesized a covalent organic polymer (COP) via dynamic covalent gel (DCG) through imine bond formation. $N, N$-Dimethyl acetamide played a significant role in the scalable synthesis of COP via gel formation. The emissive gel served as an interesting protocol for the construction of porous COP. The green emission of COP-gel was caused by an aggregationinduced emission (AIE) phenomenon, which was assisted by $\mathrm{H}$-bonding and several non-covalent interactions. On the other hand, the disperse solution of COP in DMA showed bluishwhite light emission. The degree of aggregation was important in determining the emission colour of COP in gel and solution states. The dried COP had the ability to easily adsorb aniline molecules into its cavities and separate them from binary mixtures of aniline/nitrobenzene and aniline/water. Our findings represent a significant advancement in the development of functionalized COP with excellent scalability under ambient conditions, as well as significant physical insights into the separation of aniline from miscible binary solvent mixtures.

\section{Conflicts of interest}

There are no conflicts to declare.

\section{Acknowledgements}

The authors gratefully acknowledge CSIR, Government of India (Project No. 01(2936)/18/EMR-II), for financial support and SIC, IIT Indore, for providing the required instrumental facilities. SM thanks IIT Indore, TG acknowledges DST Inspire Fellowship, Government of India, and AS acknowledges CSIR, Government of India, for their doctoral fellowship.

\section{Notes and references}

1 P. Puthiaraj, Y.-R. Lee, S. Zhang and W.-S. Ahn, J. Mater. Chem. A, 2016, 4, 16288-16311.

2 V. S. Vyas, F. Haase, L. Stegbauer, G. Savasci, F. Podjaski, C. Ochsenfeld and B. V. Lotsch, Nat. Commun., 2015, 6, 8508 .

3 P. Wang, F. Zhou, C. Zhang, S.-Y. Yin, L. Teng, L. Chen, X.-X. Hu, H. W. Liu, X. Yin and X.-B. Zhang, Chem. Sci., 2018, 9, 8402-8408.

4 Z. Miao, G. Liu, Y. Cui, Z. Liu, J. Li, F. Han, Y. Liu, X. Sun, X. Gong, Y. Zhai, Y. Zhao and Y. Zeng, Angew. Chem., Int. Ed., 2019, 58, 4906-4910.

5 C. Qian, Q.-Y. Qi, G.-F. Jiang, F.-Z. Cui, Y. Tian and X. Zhao, J. Am. Chem. Soc., 2017, 139, 6736-6743.

6 W. Luo, Y. Zhu, J. Zhang, J. He, Z. Chi, P. W. Miller, L. Chen and C.-Y. Su, Chem. Commun., 2014, 50, 11942-11945.

7 H. Zhong, Y. Gong, W. Liu, B. Zhang, S. Hu and R. Wang, Dalton Trans., 2019, 48, 2345-2351.

8 P. Song, Z. Zhang, L. Yu, P. Wang, Q. Wang and Y. Chen, New. J. Chem., 2020, 44, 8572-8577.

9 B. Mondal, D. Bairagi, N. Nandi, B. Hansda, K. S. Das, C. J. C. Edwards-Gayle, V. Castelletto, I. W. Hamley and A. Banerjee, Langmuir, 2020, 36, 12942-12953.

10 S. K. Nandi, S. Kumar, S. Roy Chowdhury, I.-O. J. Ibukun and D. Haldar, ChemistrySelect, 2021, 6, 4448-4455.

11 S. Das, P. Heasman, T. Ben and S. Qiu, Chem. Rev., 2017, 117, 1515-1563.

12 L. Tan and B. Tan, Chem. Soc. Rev., 2017, 46, 3322-3356.

13 X. Li, L. Jin, L. Huang, X. Ge, H. Deng, H. Wang, Y. Li, L. Chai and S. Ma, J. Environ. Chem. Eng., 2021, 9, 106357.

14 P. Samanta, A. V. Desai, S. Let and S. K. Ghosh, ACS Sustainable Chem. Eng., 2019, 7, 7456-7478.

15 K. Dey, S. H. Kunjattu, A. M. Chahande and R. Banerjee, Angew. Chem., Int. Ed., 2020, 59, 1161-1165.

16 W. Wang, M. Zhou and D. Yuan, J. Mater. Chem. A, 2017, 5, 1334-1347.

17 Q. Chen, M. Luo, P. Hammershøj, D. Zhou, Y. Han, B. W. Laursen, C.-G. Yan and B.-H. Han, J. Am. Chem. Soc., 2012, 134, 6084-6087.

18 Y. Yuan, F. Sun, L. Li, P. Cui and G. Zhu, Nat. Commun., 2014, 5, 4260.

19 G. He, R. Zhang and Z. Jiang, Acc. Mater. Res., 2021, 2, 630-643.

20 S. Yuan, X. Li, J. Zhu, G. Zhang, P. V. Puyvelde and B. V. Bruggen, Chem. Soc. Rev., 2019, 48, 2665-2681. 
21 S. Zhang, S. Zhao, X. Jing, Z. Niu and X. Feng, Org. Chem. Front., 2021, 8, 3943-3967.

22 J. Liu, G. Han, D. Zhao, K. Lu, J. Gao and T.-S. Chung, Sci. Adv., 2020, 6, 1110.

23 M. Ulbricht, Curr. Opin. Chem. Eng., 2020, 28, 60-65.

24 D.-W. Gao, Q. Hu, H. Pan, J. Jiang and P. Wang, Bioresour. Technol., 2015, 193, 507-512.

25 F. An, X. Feng and B. Gao, J. Hazard. Mater., 2010, 178, 499-504.

26 K. Yang, W. Wu, Q. Jing and L. Zhu, Environ. Sci. Technol., 2008, 42, 7931-7936.

27 Y. Zhou, X. Gu, R. Zhang and J. Lu, Ind. Eng. Chem. Res., 2014, 53, 887-894.

28 Y. Chen, B. Wang, X. Wang, L.-H. Xie, J. Li, Y. Xie and J.-R. Li, ACS Appl. Mater. Interfaces, 2017, 9, 27027-27035.

29 S. H. Gheewala and A. P. Annachhatre, Water Sci. Technol., 1997, 36, 53-58.

30 P. G. Jessop, L. Phan, A. Carrier, S. Robinson, C. J. Durr and J. R. Harjani, Green Chem., 2010, 12, 809-814.

31 P. G. Jessop, S. M. Mercer and D. J. Heldebrant, Energy Environ. Sci., 2012, 5, 7240-7253.

32 W. Li, B. Xu, Z. Lei and C. Dai, Chem. Eng. Process., 2018, 126, 81-89.

33 J. Gu, X. You, C. Tao, J. Li, W. Shen and J. Li, Chem. Eng. Res. Des., 2018, 133, 303-313.

34 W. Ma, J. Sun, W. Hongpu and R. Wang, Ind. Eng. Chem., 2010, 39, 781-782.

35 S. Maiti, B. Mandal, M. Sharma, S. Mukherjee and A. K. Das, Chem. Commun., 2020, 56, 9348-9351.

36 F. J. U.-Romo, C. J. Doonan, H. Furukawa, K. Oisaki and O. M. Yaghi, J. Am. Chem. Soc., 2011, 133, 11478-11481.

37 S. Maiti, A. Roy Chowdhury and A. K. Das, ChemNanoMat, 2019, 6, 99-106.
38 A. Roy Chowdhury, S. Maiti, A. Mondal and A. K. Das, J. Phys. Chem. C, 2020, 124, 7835-7843.

39 S. Dalapati, E. Jin, M. Addicoat, T. Heine and D. Jiang, J. Am. Chem. Soc., 2016, 138, 5797-5800.

40 D. Wang, S.-M. Li, Y.-F. Li, X.-J. Zheng and L.-P. Jin, Dalton Trans., 2016, 45, 8316-8319.

41 H.-Q. Yin, F. Yin and X.-B. Yin, Chem. Sci., 2019, 10, 11103-11109.

42 S. Riebe, C. Vallet, F. V. Vight, D. G. Abradelo, C. Wölper, P.-D. C. A. Strassert, G. Jansen, S. Knauer and J. Voskuhl, Chem. - Eur. J., 2017, 23, 13660-13668.

43 F.-Z. Cui, J.-J. Xie, S.-Y. Jiang, S.-X. Gan, D.-L. Ma, R.-R. Liang, G.-F. Jiang and X. Zhao, Chem. Commun., 2019, 55, 4550-4553.

44 S. Feng, S. Gong and G. Feng, Chem. Commun., 2020, 56, 2511-2513.

45 Y. Liu, Z. Qiu, H. Zhong, X. Zhao, W. Huang and X. Xing, RSC Adv., 2020, 10, 12953-12961.

46 S. Ravi, P. Puthiaraj, K. Yu and W.-S. Ahn, ACS Appl. Mater. Interfaces, 2019, 11, 11488-11497.

47 L. Deng, X. Kang, T. Quan, L. Yang, S. Liu, K. Zhang, M. Gao, Z. Xia and D. Gao, ACS Appl. Mater. Interfaces, 2021, 13, 33449-33463.

48 J.-X. Zhou, X.-S. Luo, X. Liu, Y. Qiao, P. Wang, D. Mecerreyes, N. Bogliotti, S.-L. Chen and M.-H. Huang, J. Mater. Chem. A, 2018, 6, 5608-5612.

49 Y. Liu, X. Fan, X. Jia, B. Zhang, H. Zhang, A. Zhang and Q. Zhang, J. Mater. Sci., 2016, 51, 8579-8592.

50 A. R. A. Hamid, R. Mhanna, R. Lefort, A. Ghoufi, C. AlbaSimionesco, B. Frick and D. Morineau, J. Phys. Chem. C, 2016, 120, 9245-9252.

51 C. A. Hunter, J. Mol. Biol., 1993, 230, 1025-1054. 